\title{
Effect of dosing frequency of teriparatide (PTH 1-34) on bone formation in rats: comparison of bone metabolism marker levels
}

\author{
Atsushi Watanabe ${ }^{1,6}$, Kazuyuki Tsurui $^{1}$, Shigeki Yoneyama ${ }^{2}$, Hijiri Iwata ${ }^{3}$, Takayuki Anzai ${ }^{4}$, \\ Christopher Jerome ${ }^{5}$ and Dai Nakae ${ }^{6}$ \\ ${ }^{1}$ Pharmaceuticals Research Center, Asahi Kasei Pharma Corporation, \\ 632-1 Mifuku, Izunokuni-shi, Shizuoka 410-2321, Japan \\ ${ }_{2}^{2}$ Ina Research Inc., 2148-188 Nishiminowa, Ina-shi,Nagano-ken 399-4501, Japan \\ ${ }^{3}$ LunaPath LLC Laboratory of Toxicologic Pathology, \\ 3-5-1 SLCBT-LUNA, Aoihigashi, Naka-ku, Hamamatsu-shi, 433-8114, Japan \\ ${ }^{4}$ Showa University School of Medicine, 1-5-8 Hatanodai, Shinagawa-ku, Tokyo 142-8555 Japan \\ ${ }^{5}$ Wake Forest University, 575 N. Patterson Ave., Suite 550, Winston-Salem, NC 27101 USA \\ ${ }^{6}$ Tokyo University of Agriculture, 1-1-1 Sakuragaoka, Setagaya, Tokyo 156-8502, Japan
}

(Received February 2, 2018; Accepted April 27, 2018)

\begin{abstract}
Teriparatide, a drug used in the treatment of osteoporosis, was administered to rats subcutaneously for the duration of 3 months, at a frequency of either once weekly or once daily to demonstrate the varying levels of anabolic action the drug can have on bone depending on the dosing frequency. The levels of biomarkers in the blood were compared and found to vary in osteocalcin (OC), a biomarker of bone formation, and cross-linked N-telopeptide of type 1 collagen (NTx), a biomarker of bone resorption, according to the dosing frequency. In the once-weekly regimen, teriparatide did not affect NTx levels at any of the doses studied, while OC levels increased with dose, peaking at $72 \mathrm{hr}$, then returning to normal before the next injection (after 1 week). Bone mineral density (BMD) levels increased moderately with no difference between doses. This was thought to result from the steady state achieved following increases in bone formation and bone absorption. In the once-daily dosing regimen, meanwhile, NTx levels increased with dose, and OC levels were markedly higher when compared to those with the onceweekly dosing. BMD levels were higher than those with the once-weekly dosing, but with no difference between doses. This was considered a result of unlimited, excessive increases in bone formation due to daily administration of the drug. These results suggest that teriparatide promotes normal bone metabolism ("stationary mini-modeling") when administered once weekly, and has an anabolic action with high metabolic turnover ("high-turnover remodeling") when administered once daily.
\end{abstract}

Key words: PTH, Teriparatide, Rat, Bone Biomarker, BMD, Osteoporosis

\section{INTRODUCTION}

Currently, several medications are available for the treatment of postmenopausal osteoporosis. In medical settings, bisphosphonate bone resorption inhibitors are often used as first-line drugs (Suzuki et al., 2014). Bone resorption inhibitors such as bisphosphonates, receptor activator of nuclear factor kappa-B ligand (RANKL) antibodies, and selective estrogen receptor modulators (SERMs) inhibit osteoclasts from being active and reduce the number of cells, thereby reducing bone turnover and suppressing bone resorption to prevent the worsening of osteoporosis. However, these drugs do not have effects on the promotion of bone formation. Use of these drugs may increase bone mineral density (BMD) or the amount of bone mass per unit volume and reduce bone fractures in the short term; however, they inhibit normal bone metabolism (Feng and McDonald, 2011; Iizuka and Matsukawa, 2008). Furthermore, continued intake of bone resorption inhibitors and suppression of bone turnover for extended periods of time may result in increased bone age, causing bone quality to deteriorate, and making bones more prone 
to fracture (i.e., osteoporosis) (Mashiba et al., 2001; Stepan et al., 2007; Whyte et al., 2003). In other words, although bone resorption inhibitors clearly reduce the risk of bone fracture, their effects are limited to partial reduction of bone fracture risks and potentially long-term loss of bone strength. In this light, an FDA advisory committee recently made recommendations concerning the longterm use of bone resorption inhibitors, including a 5-year limitation on the use of bisphosphonate in long-term therapy (Diab and Watts, 2013). The same recommendations are made in the AACE guidelines (Watts et al., 2010).

Teriparatide (PTH 1-34), meanwhile, has breakthrough pharmacological properties and can be used to treat osteoporosis by adding new bone. It can stimulate new bone formation, and is suitable for treating patients with osteoporosis who are at a high risk of fracture. Currently, PTH 1-34 is available as $20 \mu \mathrm{g}$ once-daily and $56.5 \mu \mathrm{g}$ once-weekly formulations. Both formulations are subject to restrictions on duration of administration, based on rat carcinogenicity studies that suggest once-daily administration may cause osteosarcoma due to excessive bone formation (Watanabe et al., 2012; Vahle et $a l ., 2002,2004)$. In addition, an early report (Subbiah et al., 2010) indicates the possible association of the once-daily PTH 1-34 with the development of osteosarcoma among patients. However, there has also been a report of patients developing osteosarcoma while not using PTH 1-34 (Andrews et al., 2012). Meanwhile, the fact that osteosarcoma did not occur with a once-weekly low-dose treatment in a rat carcinogenicity study suggests that PTH 1-34 was used at a non-carcinogenic dose (Watanabe et al., 2012). These results suggest that reduced dosing frequency of PTH 1-34 may mitigate the risk of osteosarcoma. A recent report from a study in which different dosing frequencies of PTH 1-34 were examined in rats found differences in how levels of different markers of bone formation changed with varying dosing frequency (Yamamoto et al., 2016). Studies have also been conducted in human patients with osteoporosis to examine changes in biomarker levels with once-daily (Forteo) and once-weekly (Teribone) administration (Finkelstein et al., 2010; Shiraki et al., 2013).

In response to the demand for the accumulation of academic data to support the long-term use of PTH 1-34, and with the hope that differences in bone formation may be key in explaining the mechanism of development of osteosarcoma, the authors conducted the present study in rats to compare the once-weekly and once-daily regimens, which represent the two dosing frequencies that are used in clinical settings, with a focus on the biomarkers that are involved in bone formation.

\section{MATERIALS AND METHODS}

\section{Animals and housing conditions Animals}

Male Crl:CD(SD) rats were obtained from Charles River Laboratories Japan, Inc. (Atsugi Breeding Center, Kanagawa, Japan). Animals were individually housed in stainless-steel wire cages. They were placed in a breeding room with a temperature of $21.0-25.0^{\circ} \mathrm{C}$, humidity of $40.0-70.0 \%, 12 \mathrm{hr} /$ day of light, given free access to solid rat food (CRF-1, Oriental Yeast Co., Ltd., Tokyo, Japan), and drinking water.

This study was conducted in compliance with the Act on Welfare and Management of Animals of Japan and the Ina Research Inc. guidelines for animal experimentation, and in accordance with the study protocol that was reviewed by the Institutional Animal Care and Use Committee (IACUC) of Ina Research Inc. as the testing facility. Ina Research Inc. is an AAALAC-accredited institution.

\section{Test compound}

Chemically-synthesized PTH 1-34 acetate, an active ingredient of Teribone Inj. 56.5 $\mu \mathrm{g}$ (Asahi Kasei Pharma Corp., Tokyo, Japan), was used. Lyophilized PTH 1-34 acetate was reconstituted with saline in the morning on each day of dosing.

\section{Experimental design}

A total of 60 male $\mathrm{Crl}: \mathrm{CD}(\mathrm{SD})$ rats were divided into two groups (Table 1): 30 each for the once-weekly and once-daily regimens. Within each group, three rats were assigned to the control group, and nine were assigned to each of the three dose groups. Animals in the study compound groups received subcutaneous administration of PTH 1-34 acetate for 3 months, once a week at a dose equivalent to 75,100 , or $125.4 \mu \mathrm{g} / \mathrm{kg} /$ week of PTH $1-34$, or once a day at a dose equivalent to 5,10 , or $13.6 \mu \mathrm{g} / \mathrm{kg} /$ day of PTH 1-34.

Dose setting: To set the once-weekly doses, a preliminary toxicokinetic (TK) study was conducted in advance using a 13-week, once-weekly, subcutaneous, repeateddose design. A dose that was expected to produce approximately 25 -fold exposure, namely $125.4 \mu \mathrm{g} / \mathrm{kg} / \mathrm{week}$, was set as the high dose for the present study, and doses of 100 and $75 \mu \mathrm{g} / \mathrm{kg} /$ week, with approximately 20- and 15-fold human exposure (Watanabe et al., 2012), respectively, were set as the medium and low doses, respectively. Similarly, once-daily doses were set based on exposure levels found in a 13-week subcutaneous administration study. The high dose in the present study was 
Effect of dosing frequency of teriparatide (PTH 1-34) on bone formation in rats: comparison of bone metabolism marker levels

set at $13.6 \mu \mathrm{g} / \mathrm{kg} / \mathrm{day}$, which was found to be associated with the development of osteosarcoma in carcinogenicity studies. The medium and low doses were 10 and $5 \mu \mathrm{g} / \mathrm{kg} /$ day, which were expected to produce approximately 25- and 10-fold human exposure, respectively. Animals in the control groups were given Japanese Pharmacopoeia normal saline solution (Otsuka Pharmaceutical Factory Inc., Tokushima, Japan) in the same manner.

\section{General condition}

The general condition of the animals was observed twice a day from the day prior to start of administration to the day of autopsy.

\section{Body weight}

Body weight of the animals was measured on the day before the start of administration, then twice a week during the administration period, before injection.

\section{Toxicokinetics}

Blood was collected before and $0.25,0.5,1,2,4$, and $6 \mathrm{hr}$ after the final dose given at 3 months. Plasma concentrations of PTH 1-34 acetate were measured using a High Sensitivity Human PTH (1-34) ELISA Kit (Immutopics Inc., San Clemente, CA, USA), and the concentrations of PTH 1-34 acetate in the plasma samples were determined based on the calibration curve constructed using a PTH 1-34 reference standard (Asahi Kasei Pharma Corp.). Measured mean plasma concentration of PTH 1-34 acetate in duplicates was used as the measured concentration.

\section{BMD measurement}

Computed tomography (CT) images of the third and fourth lumbar vertebrae, right femur, and right tibia were taken before administration and at the completion of the 3-month administration period, using micro-CT (R_mCT AX, Rigaku Corp., Tokyo, Japan). BMD, in $\mathrm{mg} / \mathrm{cm}^{3}$, was calculated based on the $\mathrm{CT}$ images obtained.

\section{Measurement of bone biomarker levels}

At the completion of the 3-month administration period, blood was collected before and 6, 24, 72, and $144 \mathrm{hr}$ after the final dose for the once-weekly group, and before and 6 and $24 \mathrm{hr}$ after the final dose for the once-daily group. ELISA was performed to measure serum levels of osteocalcin (OC) and cross-linked N-telopeptide of type 1 collagen (NTx) using an osteocalcin rat ELISA system (GE Healthcare Japan Corp., Tokyo, Japan) and OSTEOMARK NTx Serum (Alere Medical Co., Ltd., Tokyo, Japan), respectively, and the OC and NTx concentrations were determined based on the calibration curves constructed using their respective reference standards. Means of duplicates were used as the measured concentrations.

\section{Histopathology}

At necropsy, the following organs from animals in the control and high-dose groups were fixed in $10 \% \mathrm{v} / \mathrm{v}$ neutral buffered formalin: liver, kidney, spleen, thyroid, parathyroid, lung, bronchus, and humeral bone marrow. Hematoxylin-eosin (HE) stained samples were created according to the standard method, and all samples were examined microscopically.

\section{Statistical analysis}

For BMD data, mean and standard deviation of BMD were determined for each of the study groups before administration and after 3 months administration, and Bartlett's test was performed for homogeneity of variance (level of significance: $5 \%$ ). Where the homogeneity of variance was established, Dunnett's test was performed to compare the mean values between the control group and each of the study compound groups. Where the homogeneity of variance was not established, the data were converted logarithmically, and Bartlett's test was performed on the converted data for the homogeneity of variance (level of significance: $5 \%$ ). If the homogeneity of variance was established at this point, Dunnett's test was performed on the converted data to examine the difference in the mean values between the control group and each of the study compound groups. If, on the contrary, the homogeneity of variance was still not established, Steel's test was performed on the original measurement data to examine the difference between the control group

Table 1. Experimental design.

\begin{tabular}{|c|c|c|c|c|}
\hline Dosing frequency & On & & & \\
\hline Administration period & $\begin{array}{c}\text { Dose } \\
(\mu \mathrm{g} / \mathrm{kg} / \text { injection })\end{array}$ & No. of animals & $\begin{array}{c}\text { Dose } \\
(\mu \mathrm{g} / \mathrm{kg} / \mathrm{day})\end{array}$ & No. of animals \\
\hline Control group & 0 & 3 & 0 & 3 \\
\hline Low dose group & 75 & 9 & 5 & 9 \\
\hline Medium dose group & 100 & 9 & 10 & 9 \\
\hline High dose group & 125.4 & 9 & 13.6 & 9 \\
\hline
\end{tabular}


Table 2. Comparison of blood parameters in weekly toxicokinetic measurements after 3-month administration for once-daily groups.

\begin{tabular}{|c|c|c|c|c|}
\hline $\begin{array}{l}\text { Group } \\
(\mu \mathrm{g} / \mathrm{kg})\end{array}$ & $\begin{array}{c}\mathrm{C}_{\max } \\
(\mathrm{pg} / \mathrm{mL})\end{array}$ & $\begin{array}{l}\mathrm{T}_{\max } \\
(\mathrm{min})\end{array}$ & $\begin{array}{c}\mathrm{AUC}_{\text {all }} \\
(\mathrm{ng} \cdot \mathrm{min} / \mathrm{mL})\end{array}$ & Safety margin* \\
\hline \multicolumn{5}{|l|}{$\overline{\text { Daily } \times 7}$} \\
\hline $35(5 \times 7)$ & 3308.8 & 15 & $900.9^{*}$ & 16.3 \\
\hline $70(10 \times 7)$ & 4200.8 & 15 & $1600.9 *$ & 29.0 \\
\hline $95.2(13.6 \times 7)$ & 7685.6 & 15 & $2088.8^{*}$ & 37.9 \\
\hline \multicolumn{5}{|l|}{ Weekly } \\
\hline 75 & 24798.2 & 30 & 1277.6 & 23.2 \\
\hline 100 & 27684.6 & 15 & 1406.1 & 25.5 \\
\hline 125.4 & 37280.0 & 15 & 1876.8 & 34.0 \\
\hline
\end{tabular}

*: AUC for the once-daily groups is multiplied $7 \mathrm{x}$ to convert to 1 -week exposure.

**: $\mathrm{AUC}_{\text {last }}$ : (results on Day 1) with $56.5 \mu \mathrm{g}$ administration (sc) in osteoporosis patients $(55.14 \mathrm{ng} \cdot \mathrm{min} / \mathrm{mL})$.

and each of the study compound groups.

For biomarker data, statistical analysis was performed using R version 3.2.3 (R Foundation for Statistical Computing, Vienna, Austria). Mixed (within/between) models were analyzed using procedure lmer in package lmertest, and non-mixed models using procedure $1 \mathrm{~m}$, in both cases using type 2 hypothesis tests. In mixed and multi-way models, there were numerous significant interaction effects among dosing regimen (weekly/daily), sample timepoint ( 1 month $/ 3$ months), dose (Control, Low, Mid, High) and sample time $(0,6,24,72$ and $144 \mathrm{hr})$. Accordingly, one-way ANOVAs were used at each level of the various factors to test the effect of dose for each dosing regimen, sample timepoint and sample time. When there was a significant effect of dose, Dunnett's test was used to compare each dose group to Control.

\section{RESULTS}

\section{Body weight, general observations of symptoms, and histopathology}

No notable change was found in general observations of symptoms, body weight, and histopathological examination of major organs.

\section{Toxicokinetics}

The blood levels of PTH 1-34 were measured in animals in the once-weekly and once-daily groups after 3 months of administration. Both groups showed elevated maximum serum concentrations and total serum concentration in a dose-dependent manner, with the time to maximum plasma concentration being between 15 and 30 minutes (Table 2).

The comparison of total exposure to PTH 1-34 per week showed that the total serum concentration calculat- ed based on the total dose given was higher with the oncedaily dose than that with the once-weekly dose (Table 2). The margin of safety (Watanabe et al., 2012) was calculated based on the serum concentrations observed with the once-weekly dosing of $56.5 \mu \mathrm{g}$ of PTH 1-34 in a clinical study in osteoporotic patients. In animals treated once weekly, this was 34.0-, 25.5-, and 23.2-fold in the highest dose group (125.4 $\mu \mathrm{g} / \mathrm{kg} /$ week), medium dose group $(100 \mu \mathrm{g} / \mathrm{kg} /$ week $)$, and lowest dose group $(75 \mu \mathrm{g} / \mathrm{kg}$ / week), respectively.

\section{BMD}

Change in BMD between measurements made pre-administration and after 3 months of treatment for the fourth and fifth lumbar vertebrae, femur and tibia and were found to be higher in the PTH 1-34 groups than in the control groups for each of the measurement sites (Fig. 1). Once-daily groups showed a marked, significant, dose-dependent increase in BMD for all measurement sites at all doses except for the fourth and fifth lumbar vertebrae and tibia treated at $5 \mu \mathrm{g} / \mathrm{kg} / \mathrm{day}$. Once-weekly groups showed a marked, significant, dose-dependent increase in BMD for femur and tibia in the $75 \mu \mathrm{g} / \mathrm{kg} /$ week group.

\section{Bone biomarkers}

\section{Osteocalcin (OC): Marker of bone formation}

In the once-weekly groups (Fig. 2), OC measurements showed generally non-significant transient decreases $6 \mathrm{hr}$ after the administration, except the decrease was statistically significant at the high dose at 3 months. Osteocalcin returned to pre-injection levels after $24 \mathrm{hr}$ in all groups and timepoints. The measurements continued to increase in the PTH groups, peaking at $72 \mathrm{hr}$, and then almost returning to pre-injection levels after $144 \mathrm{hr}$. This suggests that bone formation decreased transiently and then 
Effect of dosing frequency of teriparatide (PTH 1-34) on bone formation in rats: comparison of bone metabolism marker levels

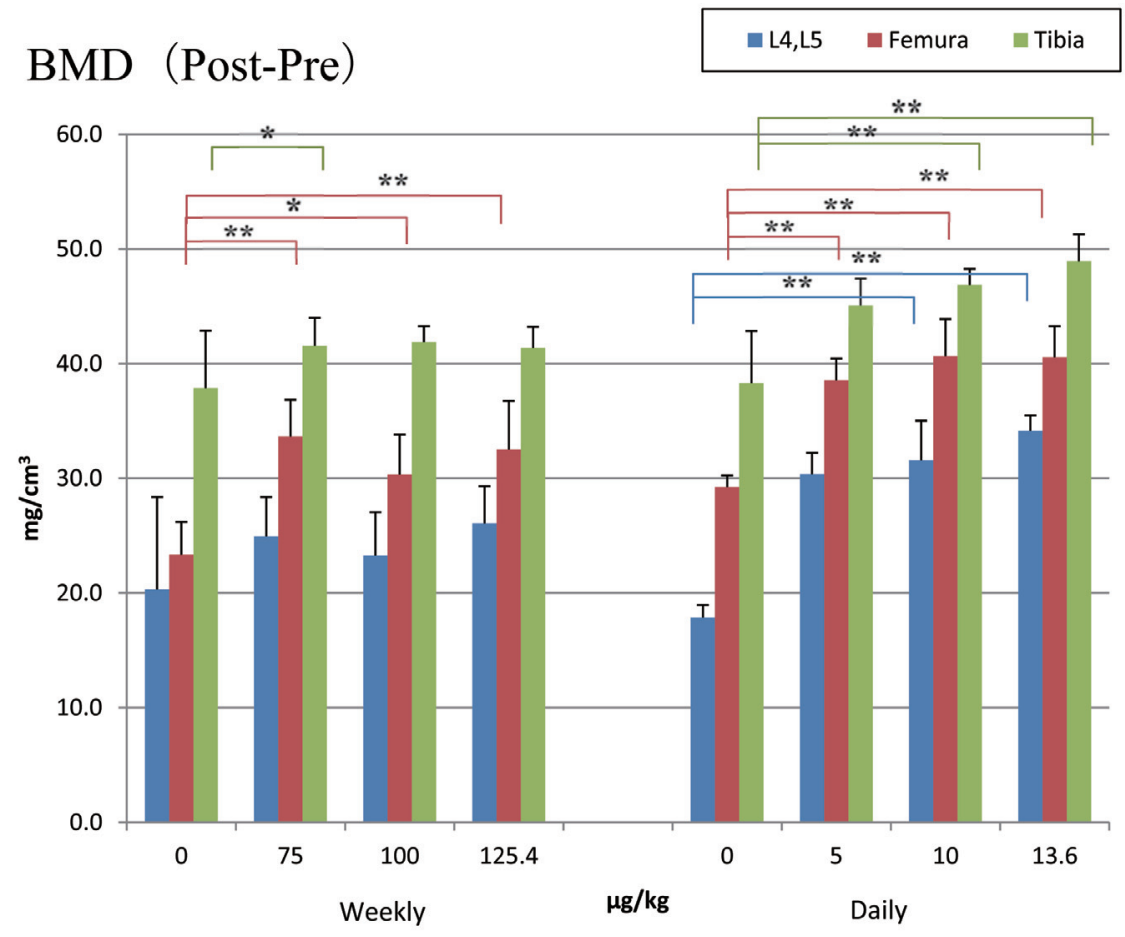

Fig. 1. Change in BMD measurement after three months' dosage. Means + SDs. *, **: Significantly different from vehicle control group at $\mathrm{P}<0.05$ and $\mathrm{P}<0.01$, respectively.

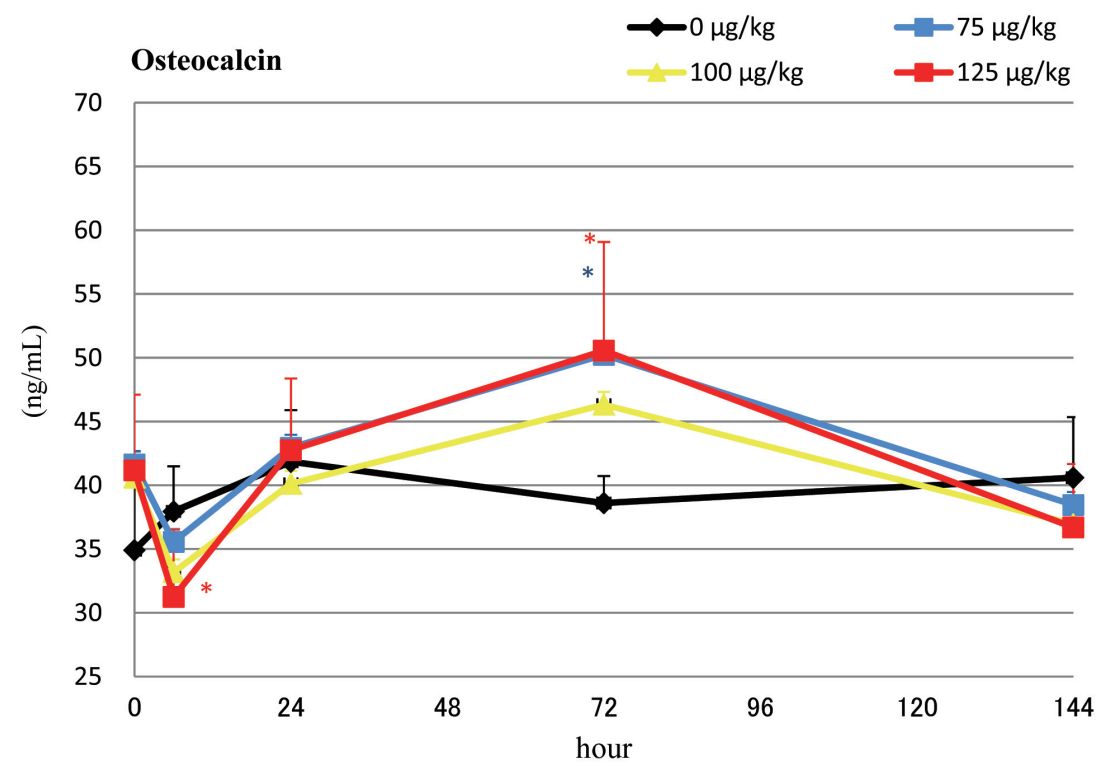

Fig. 2. Serum osteocalcin levels during the final dosing interval of the weekly dosing groups. Means + SDs. *, **: Significantly different from vehicle control group at $\mathrm{P}<0.05$ and $\mathrm{P}<0.01$, respectively. 


\section{A. Watanabe et al.}

increased several days after once-weekly administration, returning to pre-administration levels by one week.

In the once-daily groups (Fig. 3), all PTH-treated groups had significantly higher OC levels than Control at all timepoints. Control, low, and medium-dose groups showed a slight decrease at $6 \mathrm{hr}$ relative to pre-injection levels.

Cross-linked N-telopeptide of type 1 collagen (NTx): Marker of bone resorption

The once-weekly groups (Fig. 4) showed NTx levels that were not significantly different from those of the control groups.

Once-daily administration (Fig. 5), although showing little change for the first $6 \mathrm{hr}$, resulted in significantly higher levels after $24 \mathrm{hr}$ in the high- and medium-dose groups at 3 months. This suggests that once-daily administration may stimulate bone resorption slightly.

\section{DISCUSSION}

A 3-month, subcutaneous, repeated-dose study of PTH 1-34 was conducted in rats to make a comparison between once-weekly and once-daily administrations, with a focus on biomarker levels. The results showed that the serum concentrations increased with the dose for both of the dosing regimens, with the once-daily administration showing higher levels when compared in terms of total exposure per week. These high levels may be due to a general characteristic of a metabolic enzyme that is saturated when reacting with an amount of a substrate over its limit the daily administration (Gabrielsson and Weiner,
2016).

Increases in BMD, a bone biomarker that represents the amount of bone mass per unit volume, were high in all of the study compound groups i.e. once-weekly (75, 100, and $125.4 \mu \mathrm{g} / \mathrm{kg} /$ week) and once-daily $(5,10$, and $13.6 \mu \mathrm{g} / \mathrm{kg} / \mathrm{day}=35,70$, and $95.2 \mu \mathrm{g} / \mathrm{kg} /$ week), respectively, confirming the increase in bone formation.

In terms of biomarker levels, OC measurements for the once-weekly administration showed transient decreases immediately after the first injection, before increasing to peak at $72 \mathrm{hr}$ (3 days), and returning to pre-administration levels after $144 \mathrm{hr}$ (1 week). The magnitude of these changes was greater with higher doses although usually not statistically significant. With the once-daily administration, the levels were higher for all of the groups than for the control group before administration. These results suggest that the 3-month once-daily administration stimulated bone formation in a continuous and accumulative manner. As for NTx measurements, little difference was observed for the once-weekly administration compared to the control group, while the once-daily administration measurements were higher than pre-injection levels $24 \mathrm{hr}$ after administration, indicating that repeated administration increased bone resorption as well. In this research, the expression of molecular markers has not been measured, but such markers should be assessed to obtain a more precise conclusion in the future.

In summary, once-weekly administration had little effect on NTx levels while causing transiently increased bone formation based on the OC measurements, which returned to pre-administration levels before the subsequent injection. This suggests that PTH 1-34, when

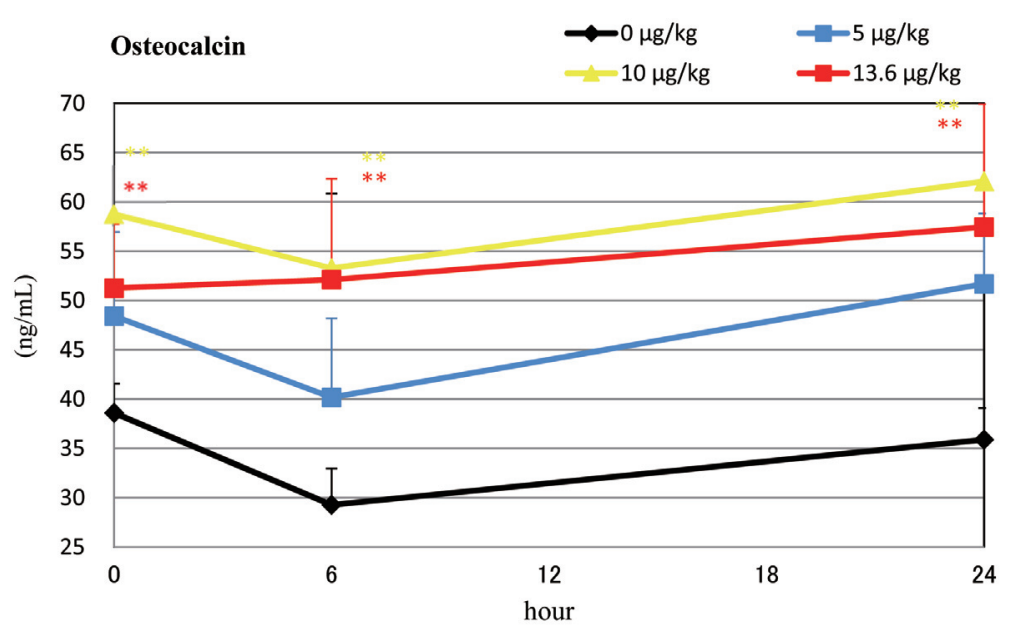

Fig. 3. Serum osteocalcin levels during the final dosing interval of the daily dosing groups. Means + SDs. *, **: Significantly different from vehicle control group at $\mathrm{P}<0.05$ and $\mathrm{P}<0.01$, respectively. 
Effect of dosing frequency of teriparatide (PTH 1-34) on bone formation in rats: comparison of bone metabolism marker levels

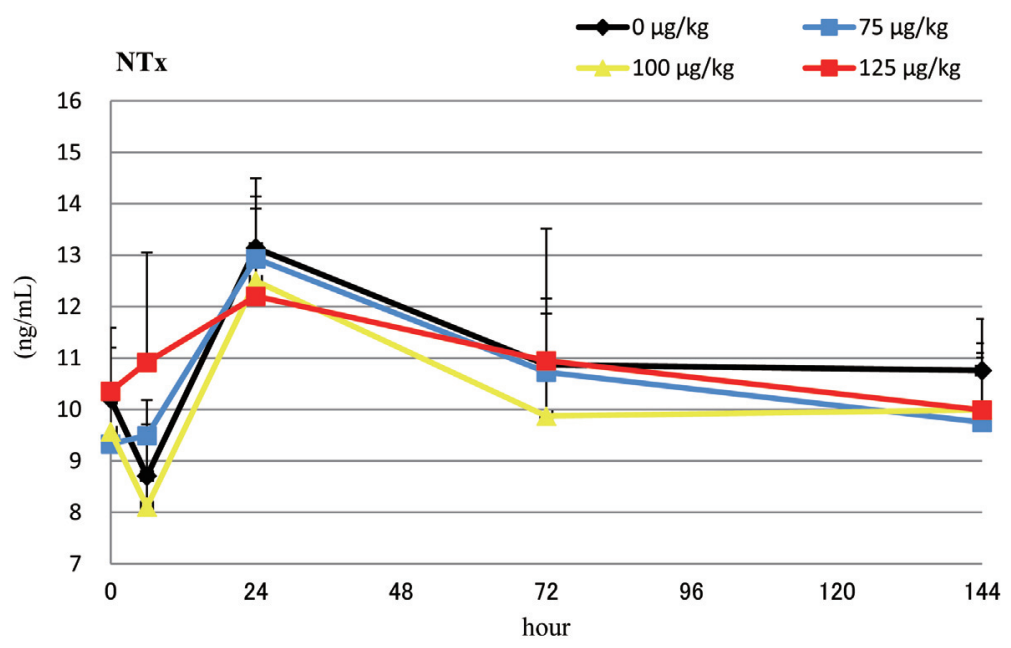

Fig. 4. Serum NTx levels during the final dosing interval of the weekly dosing groups. Means + SDs. *, **: Significantly different from vehicle control group at $\mathrm{P}<0.05$ and $\mathrm{P}<0.01$, respectively.

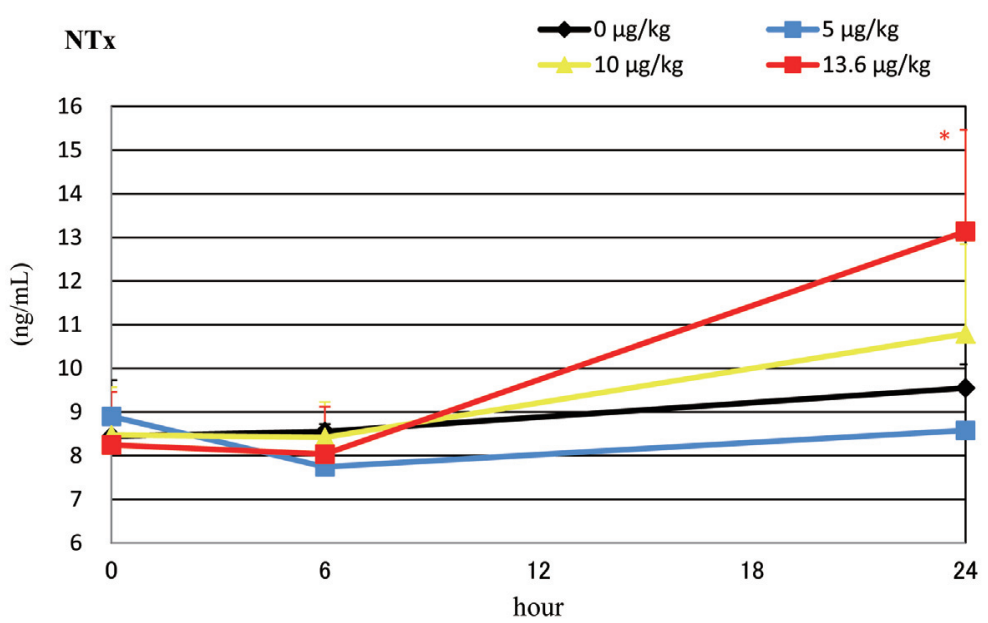

Fig. 5. Serum NTx levels during the final dosing interval of the daily dosing groups. Means + SDs. *, **: Significantly different from vehicle control group at $\mathrm{P}<0.05$ and $\mathrm{P}<0.01$, respectively.

administered once-weekly, causes increased bone formation without involving bone resorption, causing an anabolic response based on modeling. In once-daily administration, meanwhile, both OC and NTx increased with higher doses, and both bone resorption and bone formation were active, suggesting that PTH 1-34 administered daily may exert its anabolic action accompanied by high metabolic turnover ("high-turnover remodeling") (Yamamoto et al., 2016). Once weekly dosing may therefore be preferable to daily dosing, since the absence of increased bone resorption associated with the anabolic response may mitigate the risk of fracture, which has been a concern among the elderly population (Bhandari et al., 2016).

Changes in the levels of different markers (i.e., markers of BMD, bone formation, and bone resorption) observed in the present study were highly consistent with those reported in a previous clinical study (Nakamura et al., 2012). This suggests that the results obtained from rats may be readily extrapolated for predictions in humans.

In terms of carcinogenicity, bone formation of a high metabolic turnover type, such as that associated with the once-daily administration of PTH 1-34, first causes an increase in osteoclasts and promotes bone resorption, which results in the secretion of proteins such as 
transforming growth factor-beta (TGF- $\beta$ ) and insulin-like growth factor (IGF) from the bones, stimulating cell proliferation. When used in cancer patients who may have cancer cells in the bone marrow, the drug may cause such cells to release parathyroid-related protein (PTHrP), which in turn stimulates osteoblasts to express a substance known as receptor activator of nuclear factor kappa-B ligand (RANKL). Meanwhile, preosteoclasts express receptor activator of nuclear factor kappa-B (RANK) which binds to RANKL and promotes the differentiation of preosteoclasts into mature osteoclasts, thus resulting in an increased number of osteoclasts. Such a vicious cycle is considered to be involved in the development of osteosarcoma and bone metastasis (Chen et al., 2010). In other words, daily administration of PTH 1-34 may cause osteoblasts and osteoclasts to become excessively active and increase bone turnover, thus producing therapeutic effects on osteoporosis, while potentially increasing the risk of cancer exacerbation or metastasis. If used in a manner that would activate osteoblasts but not osteoclasts, such as in a once-weekly regimen, PTH 1-34 may provide a lower risk treatment option for osteoporosis.

Conflict of interest---- The authors declare that there is no conflict of interest.

\section{REFERENCES}

Andrews, E.B., Gilsenan, A.W., Midkiff, K., Sherrill, B., Wu, Y., Mann, B.H. and Masica, D. (2012): The US postmarketing surveillance study of adult osteosarcoma and teriparatide: study design and findings from the first 7 years. J. Bone Miner. Res., 27, 2429-2437.

Bhandari, M., Jin, L., See, K., Burge, R., Gilchrist, N., Witvrouw, R., Krohn, K.D., Warner, M.R., Ahmad, Q.I. and Mitlak, B. (2016): Does teriparatide improve femoral neck fracture healing: results from a randomized placebo-controlled trial. Clin. Orthop. Relat. Res., 474, 1234-1244.

Chen, Y.C., Sosnoski, D.M. and Mastro, A.M. (2010): Breast cancer metastasis to the bone: mechanisms of bone loss. Breast Cancer Res., 12, 215.

Diab, D.L. and Watts, N.B. (2013): Bisphosphonate drug holiday: who, when and how long. Ther. Adv. Musculoskelet. Dis., 5, 107-111.

Feng, X. and McDonald, J.M. (2011): Disorders of bone remodeling. Annu. Rev. Pathol., 6, 121-145.

Finkelstein, J.S., Wyland, J.J., Lee, H. and Neer, R.M. (2010): Effects of teriparatide, alendronate, or both in women with postmenopausal osteoporosis. J. Clin. Endocrinol. Metab., 95, 18381845.

Gabrielsson, J. and Weiner, D. (2016): Chapter 2.7, Nonlinear Systems - Capacity, Time, Flow and Binding. In Pharmacokinetic and Pharmacodynamic Data Analysis: Concepts and Applications, 5th edition, revised and expanded, pp.112-141, Swedish Pharmaceutical Press, Stockholm.

Iizuka, T. and Matsukawa, M. (2008): Potential excessive suppression of bone turnover with long-term oral bisphosphonate ther- apy in postmenopausal osteoporotic patients. Climacteric, 11, 287-295.

Mashiba, T., Turner, C.H., Hirano, T., Forwood, M.R., Johnston, C.C. and Burr, D.B. (2001): Effects of suppressed bone turnover by bisphosphonates on microdamage accumulation and biomechanical properties in clinically relevant skeletal sites in beagles. Bone, 28, 524-531.

Nakamura, T., Sugimoto, T., Nakano, T., Kishimoto, H., Ito, M., Fukunaga, M., Hagino, H., Sone, T., Yoshikawa, H., Nishizawa, Y., Fujita, T. and Shiraki, M. (2012): Randomized Teriparatide [human parathyroid hormone (PTH) 1-34] Once-Weekly Efficacy Research (TOWER) trial for examining the reduction in new vertebral fractures in subjects with primary osteoporosis and high fracture risk. J. Clin. Endocrinol. Metab., 97, 3097-3106.

Shiraki, M., Sugimoto, T. and Nakamura, T. (2013): Effects of a single injection of teriparatide on bone turnover markers in postmenopausal women. Osteoporos. Int., 24, 219-226.

Stepan, J.J., Burr, D.B., Pavo, I., Sipos, A., Michalska, D., Li, J., Fahrleitner-Pammer, A., Petto, H., Westmore, M., Michalsky, D., Sato, M. and Dobnig, H. (2007): Low bone mineral density is associated with bone microdamage accumulation in postmenopausal women with osteoporosis. Bone, 41, 378-385.

Subbiah, V., Madsen, V.S., Raymond, A.K., Benjamin, R.S. and Ludwig, J.A. (2010): Of mice and men: divergent risks of teriparatide-induced osteosarcoma. Osteoporos. Int., 21, 1041-1045.

Suzuki, Y., Nawata, H., Soen, S., Fujiwara, S., Nakayama, H., Tanaka, I., Ozono, K., Sagawa, A., Takayanagi, R., Tanaka, H., Miki, T., Masunari, N. and Tanaka, Y. (2014): Guidelines on the management and treatment of glucocorticoid-induced osteoporosis of the Japanese Society for Bone and Mineral Research: 2014 update. J. Bone Miner. Metab., 32, 337-350.

Vahle, J.L., Sato, M., Long, G.G., Young, J.K., Francis, P.C., Engelhardt, J.A., Westmore, M.S., Linda, Y. and Nold, J.B. (2002): Skeletal changes in rats given daily subcutaneous injections of recombinant human parathyroid hormone (1-34) for 2 years and relevance to human safety. Toxicol. Pathol., 30, 312-321.

Vahle, J.L., Long, G.G., Sandusky, G., Westmore, M., Ma, Y.L. and Sato, M. (2004): Bone neoplasms in F344 rats given teriparatide [rhPTH(1-34)] are dependent on duration of treatment and dose. Toxicol. Pathol., 32, 426-438.

Watanabe, A., Yoneyama, S., Nakajima, M., Sato, N., Takao-Kawabata, R., Isogai, Y., Sakurai-Tanikawa, A., Higuchi, K., Shimoi, A., Yamatoya, H., Yoshida, K. and Kohira, T. (2012): Osteosarcoma in Sprague-Dawley rats after long-term treatment with teriparatide (human parathyroid hormone (1-34)). J. Toxicol. Sci., 37, 617-629.

Watts, N.B., Bilezikian, J.P., Camacho, P.M., Greenspan, S.L., Harris, S.T., Hodgson, S.F., Kleerekoper, M., Luckey, M.M., McClung, M.R., Pollack, R.P. and Petak, S.M.; AACE Osteoporosis Task Force (2010): American Association of Clinical Endocrinologists Medical Guidelines for Clinical Practice for the diagnosis and treatment of postmenopausal osteoporosis. Endocr. Pract., 16 (Suppl. 3), 1-37.

Whyte, M.P., Wenkert, D., Clements, K.L., McAlister, W.H. and Mumm, S. (2003): Bisphosphonate-induced osteopetrosis. N. Engl. J. Med., 349, 457-463.

Yamamoto, T., Hasegawa, T., Sasaki, M., Hongo, H., Tsuboi, K., Shimizu, T., Ota, M., Haraguchi, M., Takahata, M., Oda, K., Luiz de Freitas, P.H., Takakura, A., Takao-Kawabata, R., Isogai, Y. and Amizuka, N. (2016): Frequency of teriparatide administration affects the histological pattern of bone formation in young adult male mice. Endocrinology, 157, 2604-2620. 